\title{
Estratégias para a biossegurança e minimização dos riscos de agravos à saude em laboratórios de um centro universitario
}

Strategies for biosafety and minimizing the risk of health problems in laboratories of a university center

\author{
Rosane Maria Kirchner, (2) Luíza Loebens ${ }^{(3)}$, Mônica Elisa Scherer ${ }^{(4)}$, Priscila Ozelame Ochôa ${ }^{(3)}$, \\ Magda Antunes de Chaves $^{(3)}$, Jaqueline Silinske ${ }^{(4)}$ Adriana Ribas Soares. \\ 'Projeto de extensão com apoio FIEX \\ ${ }^{2}$ Docente de Estatística, Doutora em Engenharia Elétrica- Métodos de Apoio à Decisão, Departamento de Zootecnia e Ciências Biológicas \\ da Universidade Federal de Santa Maria(UFSM) - CESNORS -RS- Brasil. Orientadora do Projeto de extensão. \\ ${ }^{3}$ Acadêmicas do Curso de Ciências Biológicas, Universidade Federal de Santa Maria. \\ ${ }^{4}$ Acadêmicas do Curso de Administração, Universidade Federal de Santa Maria.
}

\begin{abstract}
Resumo
Este projeto proporcionou estudar os riscos ambientais desencadeadores de agravos à saúde em laboratórios de Ensino e Pesquisa do um centro universitário e conscientizar os trabalhadores destes locais, visando desenvolver procedimentos para minimizar os riscos de acidentes pessoais e de contaminação ambiental. Foram realizadas palestras, entrevistas, distribuição de material educativo e confecção dos mapas de rico, sendo estes expostos em cada um dos laboratórios envolvidos. A partir da entrevista, verificou-se que $86,6 \%$ dos entrevistados alegaram não ter conhecimento dos temas de biossegurança e riscos a saúde e que não participaram de curso ou treinamento sobre este assunto. Em relação aos EPIs, a maioria sempre usa roupas compridas e luvas, frequentemente jaleco. 73,3\% sempre fazem uso de botinas de couro ou borracha. A elaboração do mapa de risco, de palestras, folder e a divulgação de informações sobre as EPIs serviram como um processo educativo possibilitando a socialização de conceitos sobre biossegurança bem como a conscientização dos trabalhadores sobre esta temática.
\end{abstract}

Palavras Chave: biossegurança, riscos de agravo, ambiente de trabalho, laboratórios de universidade.

\begin{abstract}
This project provided to study environmental risks triggering of aggravating health problems in Teaching and Research laboratories in a university center and to aware workers from these places in order to develop procedures to minimize the risk of personal accidents and environmental contamination. There were lectures, interviews, distribution of educational material and preparation of risk maps, which are displayed in each of the laboratories involved. From the interviews it was found that $86.6 \%$ of the interviewees claimed they had no knowledge of biosecurity issues and risks to health and they haven't attended any course or training on this subject. Regarding the PPE, most of them always wear long robes and gloves, and often smocks. $73.3 \%$ always make use of leather or rubber boots. The preparation of the risk map, lectures, brochure and information on PPE served as an educational process enabling socialization concepts and biosafety as well as awareness of workers on this issue.
\end{abstract}

Keywords: biosafety, aggravating risk, workplace, university laboratories. 


\section{INTRODUÇÃO}

$\mathrm{Na}$ sociedade, o trabalho tem sido visto não apenas como fonte de renda, mas também como meio de valorização individual e convívio social. É no ambiente de trabalho que as pessoas destinam grande parte de seu tempo, de oito horas ou mais, para tanto as condições deste espaço são essenciais para o bem-estar físico e mental. Porém, quando em circunstâncias desfavoráveis, o local de trabalho pode apresentar diversos riscos à saúde, interferindo diretamente na qualidade de vida dos trabalhadores e na biossegurança.

Biossegurança significa vida livre de perigo, genericamente, pode ser considerada como ações que contribuem para a segurança das pessoas (COSTA, 2005). Deste modo, confere-se à biossegurança como um mecanismo que tem por objetivo controlar e minimizar os riscos pertinentes à exposição, manipulação e uso de organismos vivos que podem causar efeitos desfavoráveis ao homem, bem como a animais e ao meio ambiente (COSTA, 2000).

As medidas de biossegurança são:

Conjunto de saberes direcionados para ações de prevenção, minimização ou eliminação de riscos inerentes às atividades de pesquisa, produção, ensino, desenvolvimento tecnológico e prestação de serviços, as quais possam comprometer a saúde do homem, dos animais, das plantas e do meio ambiente ou a qualidade dos trabalhos desenvolvidos (BRASIL, 2005, p.11).

No ano de 1994, com a Portaria $\mathrm{n}^{\circ} .25$, de 29 de dezembro, foi instituído o Mapa de Riscos e Programa de Prevenção de Riscos Ambientais PPRA - com o intuito de melhor orientar a adoção de medidas de controle de Riscos Ambientais nos locais de trabalho.

No Brasil, a biossegurança está baseada legalmente focando os processos que abrangem organismos geneticamente modificados, conforme a Lei de Biossegurança - N. 8974 de 05 de Janeiro de 1995. Isto é, a Lei tem como principal alvo a atenção com os riscos relativos as técnicas de manipulação de organismos geneticamente modificados (BRASIL, 2002)

Com relação ao ambiente de trabalho em laboratórios, os profissionais estão constantemente sujeitos a reagentes químicos, fluidos biológicos, material possivelmente contaminado, radiação, materiais perfuro cortantes e muitos outros riscos potenciais (ARAÚJO, 2003).
Os avanços científicos e tecnológicos colocam à disposição da sociedade um número significativo de substâncias tóxicas, que podem gerar danos à saúde, ao meio ambiente e se constituir em um problema de saúde pública (STUMM, 2010).

Neste contexto, elaborou-se um projeto de extensão sobre "Mapeamentos dos riscos ambientais em Laboratórios de Ensino e Pesquisa em um Centro Universitário da Região Norte do Rio Grande do Sul". Este teve por objetivo estudar os riscos ambientais desencadeadores de agravos à saúde dentro dos laboratórios de ensino e pesquisa do centro educacional em questão e conscientizar os trabalhadores destes locais, visando desenvolver procedimentos para minimizar os riscos de acidentes pessoais e de contaminação ambiental.

\section{MÉTODO}

O projeto foi desenvolvido junto aos colaboradores terceirizados que realizam a limpeza e manutenção dos ambientes laboratoriais do centro universitário da região norte do RS, considerando a carência de uma organização formal nas áreas de segurança, saúde e trabalho. Contou com medidas de promoção de educação em Ciências, mais especificamente em Biossegurança, gerando a capacitação dos sujeitos envolvidos com o trabalho nos laboratórios de pesquisa e ensino acerca dos riscos eminentes a este ambiente. Para tanto primeiramente realizaram-se reuniões de esclarecimento sobre o tema proposto para os trabalhadores envolvidos no funcionamento dos laboratórios a seguir foi apurada a percepção dos envolvidos sobre a temática em estudo. Esta foi realizada por meio de um questionário aplicado a 15 funcionários que atuam nos laboratórios da Universidade, no mês de maio de 2012. O questionário compreendeu questões sócio-demográficas, questões relacionadas ao tema de biossegurança e agravos à saúde, bem como, a frequência de exposição a riscos e agravos e a utilização de EPIs pelos entrevistados Os dados foram analisados com o software SPSS utilizando a estatística descritiva.

A seguir desenvolveu-se o mapeamento dos riscos ambientais nos laboratórios do Centro Educacional e foram realizadas ações de educação em Ciências Biológicas, mais especificamente em Biossegurança, relacionando temas como boas práticas em laboratórios, riscos ambientais e uso de equipamentos de proteção individual e coletiva. Para a representação gráfica dos riscos ambientais por meio dos mapas de riscos, utilizou-se o software AutoCAD. 
Para uma melhor compreensão os resultados foram apresentados na forma de palestras e desenvolveu-se material de educação e divulgação.

\section{RESULTADOS E DISCUSSÃO}

Durante a explanação dos objetivos do projeto e dos esclarecimentos sobre o tema proposto, os colaboradores terceirizados apresentaram interesse, curiosidade e participação. A seguir estes foram convidados a responder um questionário para verificar a percepção dos mesmos sobre a temática. Os resultados são apresentados a seguir.

Tabela 1 - Conhecimento e importância dos temas de Biossegurança e riscos de agravos à saúde dos profissionais que atuam nos laboratórios do centro universitário - Maio/2012

\begin{tabular}{lcc}
\hline \multicolumn{1}{c}{ Conhecimento/importância } & $\mathrm{n}$ & $\%$ \\
\hline Conhecimento sobre Biossegurança & 2 & 13,3 \\
Participação em curso/treinamento sobre Biossegurança & 2 & 13,3 \\
Importância de explicação sobre riscos de agravos à saúde & 15 & 100,0 \\
Interesse em capacitação sobre riscos de agravos à saúde & 15 & 100,0 \\
\hline
\end{tabular}

Tabela 2 - Frequência de riscos de agravos à saúde - Profissionais que atuam nos laboratórios do centro universitário sobre os temas Biossegurança e riscos de agravos à saúde - Maio/2012

\begin{tabular}{l|c|c|c|c}
\hline \multirow{2}{*}{ Riscos de agravos à saúde } & \multicolumn{3}{|c}{ Frequência } \\
\cline { 2 - 5 } Exposição a substâncias químicas nocivas. & Sempre & As vezes & Raramente & Nunca \\
Ruídos e vibração. & $\mathbf{5 ( 3 3 , 3 )}$ & $\mathbf{7 ( 4 6 , 7 )}$ & $1(6,7)$ & $2(13,3)$ \\
Agentes biológicos patogênicos como fungos, & $3(20,0)$ & $5(33,3)$ & $3(20,0)$ & $4(26,7)$ \\
bactérias, vírus e parasitas. & $5(33,3)$ & $1(6,7)$ & $4(26,7)$ & $5(33,3)$ \\
Temperatura, pressão e umidade anormais. & $3(20,0)$ & $1(6,7)$ & $4(26,7)$ & $7(46,7)$ \\
Radiação. & $3(20,0)$ & $3(20,0)$ & - & $9(60,0)$ \\
Trabalhos repetitivos e desconfortáveis. & $\mathbf{6 ( 4 0 , 0 )}$ & $\mathbf{7 ( 4 6 , 7 )}$ & $1(6,7)$ & $1(6,7)$ \\
Contato com fluidos biológicos como urina, & $\mathbf{7 ( 4 6 , 7 )}$ & $\mathbf{5 ( 3 3 , 3 )}$ & $2(13,3)$ & $1(6,7)$ \\
sangue e outras secreções corporais. & $\mathbf{5 ( 3 3 , 3 )}$ & $\mathbf{9 ( 6 0 , 0 )}$ & $1(6,7)$ & - \\
Postura inadequada durante o trabalho. & $2(13,3)$ & $3(20,0)$ & $2(13,3)$ & $8(53,3)$ \\
Animais peçonhentos. & $2(13,3)$ & $2(13,3)$ & $4(26,7)$ & $7(46,7)$ \\
Armazenamento inadequado de produtos químicos. & $\mathbf{3 ( 2 0 , 0 )}$ & $1(6,7)$ & $3(20,0)$ & $8(53,3)$ \\
Máquinas e equipamentos perigosos. & $2(13,3)$ & $1(6,7)$ & $3(20,0)$ & $9(60,0)$ \\
Ferramentas e instrumentos de trabalho & $3(20,0)$ & $2(13,3)$ & $1(6,7)$ & $9(60,0)$ \\
inadequados ou defeituosos. & $\mathbf{4 ( 2 6 , 7 )}$ & $2(13,3)$ & $1(6,7)$ & $8(53,3)$ \\
Probabilidade de incêndio. & $3(20,0)$ & $4(26,7)$ & $5(33,3)$ & $3(20,0)$ \\
Inadequada eliminação e tratamento do lixo. & $\mathbf{7 ( 4 6 , 7 )}$ & $\mathbf{7 ( 4 6 , 7 )}$ & - & $1(6,7)$ \\
Situações causadoras de estresse físico ou \\
psicológico.
\end{tabular}


Observou-se nos dados sociodemográficos que $60 \%$ dos entrevistados são do sexo feminino, $73,4 \%$ com idade entre 20 a 40 anos e $26,7 \%$ com 40 anos ou mais. Em relação à escolaridade, 53,3\% tem ensino médio completo, $40 \%$ ensino fundamental completo e $6,7 \%$ ensino superior completo. Quanto à função, 33,3\% são auxiliares de limpeza, $33,3 \%$ serventes, $20 \%$ profissionais de serviços gerais e $13,3 \%$ profissionais da manutenção.

Quando indagados sobre o conhecimento dos temas de biossegurança e riscos a saúde, $86,6 \%$ alegaram não saber sobre o mesmo e que nunca participaram de curso ou treinamento sobre o assunto. Todos os entrevistados apresentaram interesse em participar de uma capacitação sobre riscos de agravos à saúde do trabalhador além de considerar importante uma explicação sobre o tema (Tabela 1). $\mathrm{O}$ alto índice de desconhecimento sobre o assunto confirma a necessidade de se implantar uma cultura de valorização do homem e de sua qualidade de vida, o que, para Moreno et al.(2008) dependerá não só da intensificação em educação e treinamento mas também na conscientização dos profissionais de saúde e de todos os envolvidos na área por meio de uma educação continuada.

O trabalho em laboratórios envolve riscos gerais e outros específicos a cada área de atividade, podendo ser classificados em riscos químicos, físicos, biológicos, acidentes e ergonômicos, conforme a Portaria do Ministério do Trabalho, MTb $n^{\circ} 3.214$, de 8/6/1978 (BRASIL, 1978).

Os entrevistados, quando questionados sobre a frequência de riscos de agravos a saúde em que estão expostos (Tabela 2), apontaram com maior percentual que ocorre "sempre" ou "às vezes", a exposição às substâncias químicas nocivas, ruídos e vibração, trabalhos repetitivos e desconfortáveis, contato com fluidos biológicos como urina, sangue e outras secreções corporais, postura inadequada durante o trabalho, piso escorregadio e escadas sem proteção.

Com relação ao uso de EPIs (Equipamentos de Proteção Individual) os resultados foram satisfatórios, pois mais de $86 \%$ dos entrevistados afirmaram sempre usar roupas compridas e $80 \%$ usam jaleco frequentemente. Todos utilizam as luvas classificando a frequência como "às vezes" ou "sempre" e 73,3\% "sempre" usam botinas de couro ou borracha (Tabela 3).

O cuidado e o uso adequado dos EPIs em ambientes laboratoriais é de suma importância, uma vez que os profissionais, segundo Lubarino (2009), quando atuam com negligência, ausência ou uso inadequado de Equipamentos de Proteção Individual (EPI) ou Equipamentos de Proteção Coletiva (EPC) estão colocando as pessoas que frequentam e/ou realizam suas atividades nestes ambientes, à exposição de vulnerabilidade a riscos ambientais.

Cabe resaltar que este projeto teve como metodologia fundamental a implementação de medidas de biossegurança nos laboratórios de Ensino e Pesquisa do Centro Educacional, por meio do mapeamento dos riscos ambientais e representação gráfica destes por intermédio dos mapas de risco. Os riscos ambientais a que estão expostos os sujeitos que atuam nos laboratórios sediados na instituição de ensino foram mapeados considerando principalmente riscos classificados como: químicos, biológicos, ergonômicos e de acidentes (BRASIL, 1978).

A análise de risco é uma metodologia

Tabela 3 - Frequência do uso de Equipamentos de Proteção Individual (EPI)

Profissionais que atuam nos laboratórios centro universitário sobre os temas Biossegurança e riscos de agravos à saúde - Maio/2012

\begin{tabular}{l|c|c|c|c}
\hline \multirow{2}{*}{ Equipamentos de Proteção Individual } & \multicolumn{4}{c}{ Frequência } \\
\cline { 2 - 5 } & Sempre & Às vezes & Raramente & Nunca \\
\hline Roupas compridas. & $13(86,7)$ & - & $1(6,7)$ & $1(6,7)$ \\
Jaleco/avental. & $12(80,0)$ & $1(6,7)$ & $1(6,7)$ & $1(6,7)$ \\
Luvas. & $9(60,0)$ & $6(40,0)$ & - & - \\
Botinas de couro/borracha. & $11(73,3)$ & $3(20,0)$ & - & $1(6,7)$ \\
Máscara. & $1(6,7)$ & $4(26,7)$ & $4(26,7)$ & $6(40,0)$ \\
Óculos de proteção. & $3(20,0)$ & - & $2(13,3)$ & $10(66,7)$ \\
Protetor auditivo. & $1(6,7)$ & $3(20,0)$ & - & $11(73,3)$ \\
Chapéu. & $1(6,7)$ & $3(20,0)$ & - & $11(73,3)$ \\
Protetor solar. & - & $2(13,3)$ & - & $13(86,7)$ \\
\hline
\end{tabular}


científica que auxilia na busca sistematizada de informações sobre um determinado perigo, de forma a permitir a avaliação do risco envolvido e a adoção de medidas para eliminar ou controlar o perigo detectado (LAJOLO \& NUTTI, 2003). Essa metodologia configurou-se na forma de um procedimento científico sistematizado nas etapas: 1. Avaliação do risco; 2. Gerenciamento do risco; 3. Informação do risco; 4. Desenvolvimento de métodos de biossegurança (BRASIL, 1994).

$\mathrm{Na}$ etapa de avaliação do risco, realizou-se uma visita aos laboratórios da instituição, realizando análise e caracterização da natureza quantitativa e qualitativa dos riscos ambientais. Para tanto, obteve-se informações principalmente com os técnicos de laboratórios responsáveis.

A partir dos riscos avaliados, procurou-se desenvolver medidas de regulamentação e controle dos riscos do ambiente. Nesta fase de gerenciamento do risco, a principal finalidade foi desenvolver medidas para minimização dos possíveis riscos de acidentes e agravos à saúde nos laboratórios.

A fase seguinte, de informação do risco, envolveu a representação gráfica dos riscos ambientais por meio dos mapas de riscos, representados sobre a planta baixa dos laboratórios, de acordo com a simbologia de círculos e cores (Quadro 1 e Figura 1). Na figura 2, consta um exemplo dos mapas de riscos elaborados para cada um dos laboratórios. Por fim, realizou-se a comunicação de risco aos colaboradores envolvidos com os trabalhos nos laboratórios, principalmente aqueles que sujeitos expostos aos riscos.

Quadro 1: Classificação dos Riscos ambientais de acordo com sua natureza e a padronização das cores correspondentes.

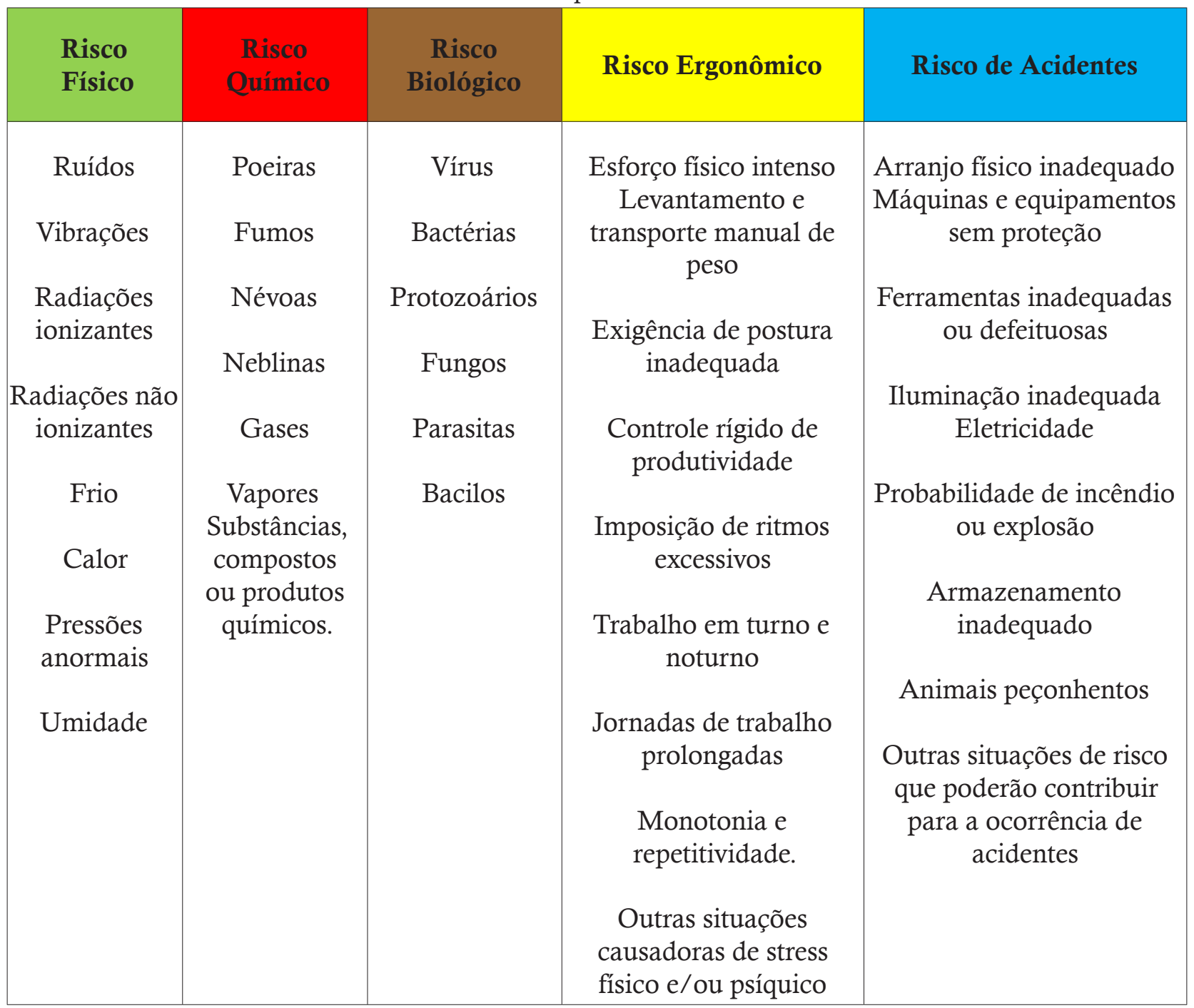

Fonte: sebrae - saúde e segurança no trabalho - dicas de prevenção de acidentes e doenças no trabalho. Brasília, 2005. 
NATUREZA DO RISCO

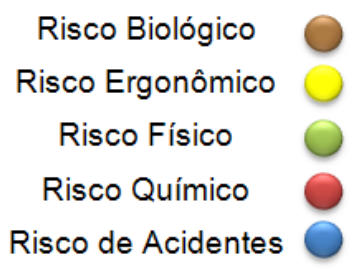

INTENSIDADE DO RISCO

Risco Mínimo

Risco Médio

Risco Máximo

Figura 1: Legenda da Natureza e Intensidade do Risco de acordo com a padronização das cores correspondentes. Fonte: SEBRAE - Saúde e Segurança no Trabalho -Dicas de prevenção de acidentes e doenças no trabalho. Brasília, 2005.

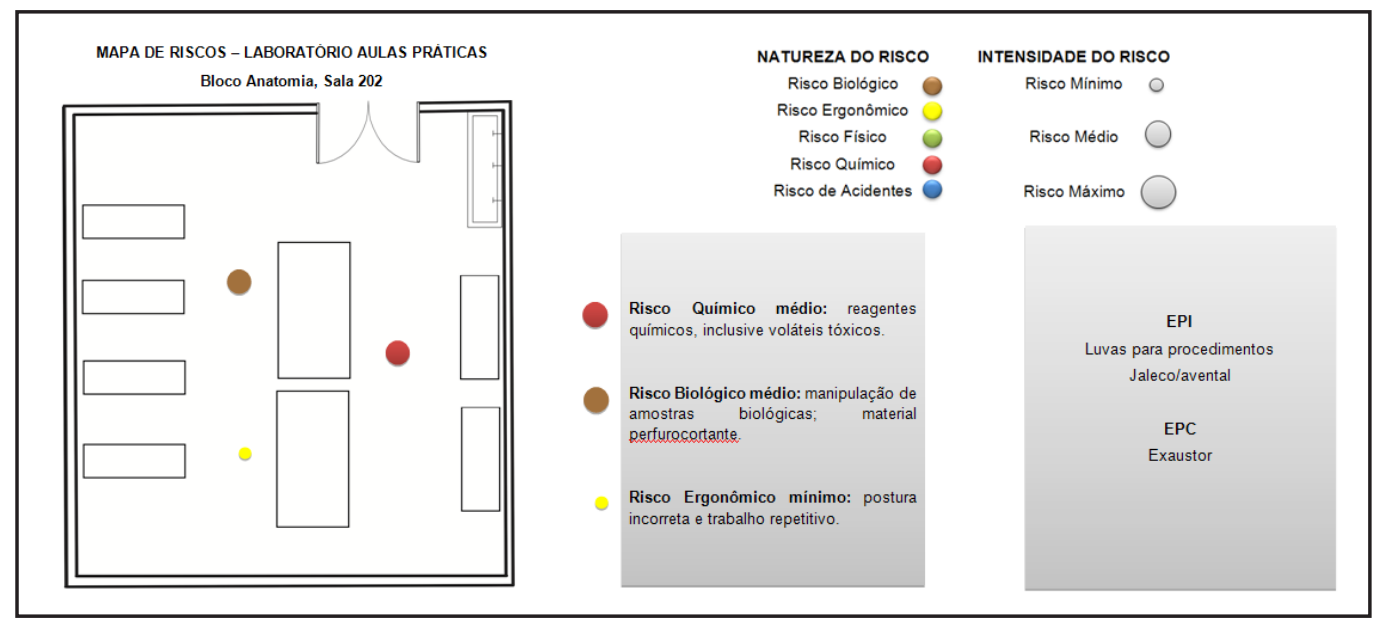

Figura 2: Exemplo dos mapas de riscos elaborados para cada um dos laboratórios.

A etapa final do mapeamento de riscos relaciona-se ao desenvolvimento de métodos de biossegurança. Para tanto, procurou-se elaborar estratégias para minimização dos acidentes, relacionadas à capacitação da população envolvida para uso de técnicas de proteção individual e coletiva. Nesse sentido, o enfoque foi para os servidores terceirizados que realizam a limpeza Realizou-se orientação relacionada à interpretação dos mapas de risco. Inicialmente havia sido proposto realizar uma reunião com todos os trabalhadores, para assim desenvolver uma palestra de capacitação em Biossegurança, contudo acabou sendo adotada uma metodologia mais flexível. Desta forma, foi confeccionado um folder para ser utilizado como material de educação e divulgação, envolvendo orientações relativas aos mapas de risco e a importância do uso dos equipamentos de proteção individual.

As "palestras" foram realizadas em grupos pequenos, em horários flexíveis, na forma de visitas aos principais laboratórios. Sendo que durante as visitas os folders foram distribuídos e procurou-se dar ênfase e demonstrar os equipamentos, reagentes e fatores que oferecem maiores riscos de agravos à saúde. Deve-se destacar que tradicionalmente os equipamentos de proteção individual são apontados como soluções altamente eficazes, e o não uso seria considerado como demonstração da irresponsabilidade e falta de consciência dos trabalhadores. Para Hökerberg et al. (2006), priorizar a identificação dos riscos pelos trabalhadores, provocar discussões sobre os geradores destes riscos, bem como, sobre o ambiente de trabalho são estratégias preventivas para reduzir os riscos identificados pelo grupo de trabalhadores.

Para o trabalhador que se expõe a situações de risco o hábito de utilizar EPIs, deve ser encarado como meio de proteção disponível em uma visão associada e sistêmica dos agravos provenientes das atividades ocupacionais (VEIGA, 2007).

\section{CONCLUSÃO}

Os resultados obtidos no questionamento evidenciaram o comportamento dos entrevistados, bem como o conhecimento sobre Biossegurança e riscos de agravos à saúde. A maioria deles declarou desconhecer a temática de biossegurança, porém todos se mostraram interessados em compreender mais sobre o assunto, por meio de palestras e capa- 
citações. E ainda, verificou-se que os mesmos têm consciência da importância do uso de EPIs, e os utilizam regularmente para sua maior proteção no ambiente de trabalho. Por fim, constatou-se que os riscos de agravos à saúde que ocorrem nos locais do estudo são principalmente riscos biológicos, riscos ergonômicos e riscos de acidentes.

A atividade de confecção dos mapas de riscos e exposição dos mesmos nos laboratórios tornou-se um processo pedagógico, tendo sido desenvolvida de maneira integrada, pois possibilitou a participação de um número considerável de colaboradores, proporcionando a socialização do saber coletivo e o desenvolvimento de soluções em biossegurança. Além disso, o folder, as palestras bem como a apresentação dos mapas de rico serviram como material educativo em biossegurança permitindo o esclarecimento a cerca da interpretação dos mesmos e informações adicionais sobre o uso de equipamentos de proteção individual.

Mediante a isso, o conjunto de ações desenvolvido proporcionou a oportunidade de socialização e democratização do conhecimento de Ciências, mais especificamente em biossegurança.

\section{REFERÊNCIAS BIBLIOGRÁFICAS}

ARAÚJO, A. K. Avaliação dos Riscos Ambientais da Divisão de Produtos do Laboratório Central de Saúde Pública do Ceará - LACEN/CE. $2003.74 \mathrm{f}$. Monografia (Especialização em Alimentos e Saúde Pública) - Universidade Federal do Ceará, Fortaleza, 2003.

BRASIL. Ministério da Saúde. Procedimentos para a manipulação de microorganismos patogênicos e/ ou recombinantes na Fiocruz. Rio de Janeiro, RJ, 2005.

BRASIL. Ministério da Ciência e Tecnologia. Cadernos de Biossegurança - Legislação, 2002.

BRASIL. Ministério do Trabalho. Portaria $n^{\circ} 25$ de 29/12/1994. Brasília, DF, 1994.

BRASIL. Diário Oficial da União. NR 9 - Programa de prevenção de riscos ambientais. 1978.

COSTA, M.A.F. Qualidade em Biossegurança. Rio de Janeiro: Qualitymark, p. 100, 2000.

COSTA, M.A.F. Construção do Conhecimento em Saúde: estudo sobre o ensino de biossegurança em cursos de nível médio da área de saúde da
Fundação Oswaldo Cruz. 2005. 154 f. Tese (Doutorado em Ensino de Biociências e Saúde) - Instituto Oswaldo Cruz, Rio de Janeiro, 2005.

HÖKERBERG, Y. H. M. et al. O processo de construção de mapas de risco em um hospital público. Ciência \& Saúde Coletiva, v. 11, n. 2, p. 503-513, 2006.

LAJOLO, F. M.; NUTTI, M. R. Transgênicos: Bases Científicas da sua segurança. São Paulo: SBAN, p. 26-27, 2003.

LUBARINO, A. E. Avaliação dos Riscos Biológicos Associados à Pesquisa de Campo e Análise de Pescados no Ambiente dos Laboratórios de Microbiologia Aplicada, Ambiental e Saúde Pública (LAMASP) e Laboratório de Qualidade dos Alimentos (LAQUA) da Universidade Estadual de Feira de Santana. 2009. 65 f. Monografia (Especialização em Biologia Celular) - Universidade Federal de Feira de Santana, Bahia, 2009.

MORENO, M. C.; ENDERS, B. C.; SIMPSON, C. A. Avaliação das capacitações de hanseníase: Opinião dos médicos e enfermeiros das equipes de saúde da família. Revista Brasileira de Enfermagem, Brasília, v. 61, p. 671- 675. 2008.

STUMM, E. M. F.; LORO, M. M.; KIRCHNER, R. M.; LIAMARA, A. Z.; UBESSI, D.; POLI, A.G.; FRANCO, M. T. Análise do uso de equipamentos de proteção individual por agricultores que utilizam agrotóxicos. Revista Técnico-Científica de Enfermagem. v. 8, n. 25, 2010.

VEIGA, M.M.; DUARTE, F.J.C.M.; MEIRELLES, L.A.; GARRIGOU, A.; BALDI, I. A contaminação por agrotóxicos e os equipamentos de proteção individual (EPIs). Revista Brasileira de Saúde Ocupacional. v. 32, n. 116, p. 57-68, 2007. 